\title{
VARIATION IN MORPHO-PHYSIOLOGICAL CHARACTERS AND YIELD COMPONENTS OF SUMMER MUNGBEAN (VIGNA RADIATA (L.) WILCZEK) VARIETIES
}

\author{
MMA Mondal ${ }^{1 *}$, MA MaleK ${ }^{2}$ ANd AB Puteh ${ }^{1}$ \\ Crop Physiology Division, Bangladesh Institute of Nuclear Agriculture, \\ Mymensingh, Bangladesh
}

Key words: Summer mungbean, Growth, Yield components, Seed yield

\begin{abstract}
Twelve modern summer mungbean cultivars were evaluated to assess morpho-physiological characters, yield attributes and seed yield. Results revealed that high yielding cultivars, in general, showed superiority in morpho-physiological characters and seed yield/plant than the low yielding cultivars. However, in case of unit area basis, result revealed that seed yield/ha was greater in low yielding cultivars than the high yielding ones, yet they produced lower seed yield/plant compared to high yielding ones due to increase number of plants per unit area and these low yielding cultivars also matured $10-15$ days earlier than high yielders. Among the cultivars, BINAmung-5 produced the highest seed yield/ha $(1711 \mathrm{~kg} / \mathrm{ha})$ with lower $\mathrm{HI}(20.0 \%)$ and took longer days to maturity (69 days). On the other hand, BARImung-6 showed second highest seed yield $(1697 \mathrm{~kg} / \mathrm{ha})$ with highest $\mathrm{HI}(32.6 \%)$ and matured earliest, took 60 days after sowing that might be fit the existing cropping pattern in Bangladesh.
\end{abstract}

The principal constraint of mungbean [Vigna radiata (L.) Wilczek] production is its low yield potential. Most of the flowers (70 - 95\%) of mungbean do not develop into mature pods (Fakir et al. 2011) indicating that potential fruit or seed number is usually much larger than the number actually produced by the plant community. The number of fruits increases after growth stage R1 (Fruit setting stage) and reaches a maximum after growth stage R5 (Seed growth stages) (Mondal et al. 2011a) but during this period the plant is still growing vegetatively. Therefore, developing reproductive sinks are competing for assimilates with vegetative sinks.

Important physiological attributes such as leaf area index (LAI), crop growth rate (CGR), net assimilation rate (NAR) and specific leaf weight (SLW) can address various constraints of a variety for increasing its productivity (Pandey et al. 1978). A plant with optimum LAI and NAR may produce higher biological yield. The capability of efficient partitioning between the vegetative and reproductive parts may produce high economic yield (Mondal et al. 2011b). The dry matter accumulation may be the highest if the LAI attains its maximum value within the shortest possible time (Mondal et al. 2011c).

In Bangladesh, Bangladesh Agricultural Research Institute (BARI) and Bangladesh Institute of Nuclear Agriculture (BINA), and Bangabundhu Sheikh Mazibur Rahman Agricultural University (BSMRAU) have developed a couple of varieties of summer mungbean, which are high yielding compared to local landrace. These varieties need to be assessed for their physiological growth and morphological maneuvering to select better one. Hence, the present research work has been designed to study morpho-physiological parameters, reproductive characters and other yield attributes responsible for higher seed yield in 12 summer mungbean varieties to select best one for countrywide cultivation.

*Author for correspondence: <mmamondal@gmail.com>. ${ }^{1}$ Department of Crop Science, Faculty of Agricuture, Universiti Putra Malaysia, 43400 UPM Serdang, Selangor Darul Ehsan, Malaysia. ${ }^{2}$ Plant Breeding Division, Bangladesh Institute of Nuclear Agriculture, Mymensingh, Bangladesh. 
Six field experiments were performed at different agro-ecological zones of Bangladesh such as Mymensingh, Magura, Chuadanga, Pabna, Natore and Rangpur districts in Kharif-I (FebruaryMay) season of 2009. Released twelve mungbean varieties were used as planting material. The name of the varieties is presented in Table 1. Seeds were sown between 05 and 17 March, 2009. A randomized complete block design with three replicates was followed. A unit plot size of $3 \mathrm{~m} \times 3$ $m$ with plant spacing of $30 \mathrm{~cm} \times 10 \mathrm{~cm}$ or $25 \mathrm{~cm} \times 10 \mathrm{~cm}$ (depending on variety) was used at all the locations. Recommended intercultural practices such as fertilization, weeding, thinning and application of pesticides were followed for proper growth and development of the plants.

The morpho-physiological parameters, yield components and seed yield/plant were recorded at Mymensingh and seed yield/plot data were recorded at all locations and converted into tons/ha. To study ontogenetic growth characteristics, a total of five harvests were made. The first crop sampling was done at 25 days after sowing (DAS) and continued at an interval of ten days up to 65 DAS i.e. till attaining physiological maturity of some varieties. From each sampling, five plants were randomly selected from second and third rows of each plot and uprooted for collecting necessary parameters. The plants were separated into roots, stems, leaves and pods, and the corresponding dry weight were recorded after oven drying at $80 \pm 2^{\circ} \mathrm{C}$ for $72 \mathrm{hrs}$. The leaf area of each sample was measured by automatic leaf area meter (Model: LICOR 3000, USA). The growth analysis like absolute growth rate was carried out following the formulae of Hunt (1978). The yield contributing characters were recorded at harvest from ten competitive plants of each plot. The seed yield was recorded from five rows of each plot $(1.50 \mathrm{~m} \times 3.0 \mathrm{~m})$ and converted into seed yield/hectare and seed weight/plant was determined by dividing the plant number. Harvest index was calculated from the collected data using formula: (economic yield/plot $\div$ biological yield/plot) $\times 100$. The collected data were analyzed statistically by using computer package programme, MSTAT-C.

Table 1. Leaf area development at different growth stages in 12 summer mungbean varieties.

\begin{tabular}{llllll}
\hline \multirow{2}{*}{ Varieties } & \multicolumn{5}{c}{ Leaf area/plant $\left(\mathrm{cm}^{2}\right)$} \\
\cline { 2 - 6 } & $25 \mathrm{DAS}$ & $35 \mathrm{DAS}$ & $45 \mathrm{DAS}$ & $55 \mathrm{DAS}$ & $65 \mathrm{DAS}$ \\
\hline BARImung-2 & $64.1 \mathrm{c}$ & $479 \mathrm{bc}$ & $748 \mathrm{~d}$ & $1212 \mathrm{c}$ & $1388 \mathrm{~d}$ \\
BARImung-3 & $42.0 \mathrm{f}$ & $546 \mathrm{a}$ & $619 \mathrm{e}$ & $841 \mathrm{~d}$ & $895 \mathrm{e}$ \\
BARImung-4 & $72.3 \mathrm{~b}$ & $473 \mathrm{c}$ & $937 \mathrm{~b}$ & $1753 \mathrm{a}$ & $2128 \mathrm{a}$ \\
BARImung-5 & $49.2 \mathrm{e}$ & $372 \mathrm{~d}$ & $538 \mathrm{fg}$ & $628 \mathrm{f}$ & $572 \mathrm{f}$ \\
BARImung-6 & $49.0 \mathrm{e}$ & $277 \mathrm{e}$ & $494 \mathrm{~g}$ & $623 \mathrm{f}$ & $580 \mathrm{f}$ \\
BINAmung-2 & $95.0 \mathrm{a}$ & $498 \mathrm{bc}$ & $999 \mathrm{ab}$ & $1243 \mathrm{c}$ & $2063 \mathrm{a}$ \\
BINAmung-5 & $92.4 \mathrm{a}$ & $521 \mathrm{ab}$ & $850 \mathrm{c}$ & $1216 \mathrm{c}$ & $1028 \mathrm{e}$ \\
BINAmung-6 & $67.4 \mathrm{bc}$ & $379 \mathrm{~d}$ & $586 \mathrm{ef}$ & $683 \mathrm{ef}$ & $580 \mathrm{f}$ \\
BINAmung-7 & $56.6 \mathrm{~d}$ & $346 \mathrm{~d}$ & $1013 \mathrm{a}$ & $1472 \mathrm{~b}$ & $1570 \mathrm{c}$ \\
BUmung-1 & $51.9 \mathrm{de}$ & $266 \mathrm{e}$ & $689 \mathrm{~d}$ & $1397 \mathrm{~b}$ & $1840 \mathrm{~b}$ \\
BUmung-2 & $53.2 \mathrm{de}$ & $282 \mathrm{e}$ & $592 \mathrm{ef}$ & $780 \mathrm{de}$ & $662 \mathrm{f}$ \\
BUmung-4 & $68.8 \mathrm{bc}$ & $345 \mathrm{~d}$ & $493 \mathrm{~g}$ & $608 \mathrm{f}$ & $613 \mathrm{f}$ \\
\hline F-test & $* *$ & $* *$ & $* *$ & $* *$ & $* *$ \\
\hline CV $(\%)$ & 4.79 & 6.38 & 5.51 & 6.72 & 8.82 \\
\hline
\end{tabular}

In a column, means followed by same letter(s) do not differ significantly at 5\% level by DMRT.

**Significant at $1 \%$ level of probability.

The leaf area (LA), total dry matter (TDM) production and absolute growth rate (AGR) of summer mungbean cultivars were differed significantly at all growth stages (Tables 1,2). The LA 
increased with age till 55 DAS followed by a decline at physiological maturity (65 DAS) in six varieties (BARImung-5, BARImung-6, BINAmung-5, BINAmung-6, BUmung-2 and BUmung-4) out of twelve due to leaf shedding (Table 1). The other six cultivars, BARImung-2, BARImung-3, BARImung-4, BARImung-6, BINAmung-2, BINAmung-7 and BUmung-1 showed increasing trend in LA till 65 DAS because of they were then at flowering and pod development stages. It is noted that BARImung-2, BARImung-4, BINAmung-2, BINAmung-5, BINAmung-7 and BUmung-1 matured nearly 72-75 DAS where as other six cultivars (BARImung-3, BARImung-5, BARImung-6, BINAmung-6, BUmung-2 and BUmung-4) matured at 60-65 DAS (Table 4). The cultivars BARImung-4, BINAmung-2, BINAmung-7 and BUmung-1 showed superiority in LA at later growth stages compared to other eight cultivars. In contrast, BARImung-5, BARImung-6, BINAmung-6, BUmung-2 and BUmung-4 showed inferiority in case of LA over its growth period and also matured 10-15 days earlier than the remainder.

Table 2. Total dry mass production and absolute growth rate at different growth stages in 12 summer mungbean varieties

\begin{tabular}{|c|c|c|c|c|c|c|c|c|c|}
\hline \multirow[b]{2}{*}{ Varieties } & \multicolumn{5}{|c|}{ Total dry mass/plant (g) } & \multicolumn{4}{|c|}{ Absolute growth rate (mg/plant/day) } \\
\hline & $\begin{array}{l}25 \\
\text { DAS }\end{array}$ & $\begin{array}{l}35 \\
\text { DAS }\end{array}$ & $\begin{array}{l}45 \\
\text { DAS }\end{array}$ & $\begin{array}{l}55 \\
\text { DAS }\end{array}$ & $\begin{array}{l}65 \\
\text { DAS }\end{array}$ & $\begin{array}{l}25-35 \\
\text { DAS }\end{array}$ & $\begin{array}{l}35-45 \\
\text { DAS }\end{array}$ & $\begin{array}{l}45-55 \\
\text { DAS }\end{array}$ & $\begin{array}{l}55-65 \\
\text { DAS }\end{array}$ \\
\hline BARImung-2 & $0.57 \mathrm{~d}$ & $3.78 \mathrm{bc}$ & $10.9 \mathrm{a}$ & $22.72 \mathrm{a}$ & $27.29 \mathrm{c}$ & $321 b$ & $683 a$ & $711 \mathrm{de}$ & $857 \mathrm{~d}$ \\
\hline BARImung-3 & $0.41 \mathrm{~h}$ & $4.34 \mathrm{a}$ & $8.77 \mathrm{cde}$ & $14.62 \mathrm{de}$ & $23.82 \mathrm{~d}$ & $376 a$ & $393 f$ & $685 \mathrm{e}$ & $1020 \mathrm{c}$ \\
\hline BARImung-4 & $0.62 \mathrm{~cd}$ & $4.14 \mathrm{ab}$ & $9.34 \mathrm{c}$ & $18.65 b$ & $32.63 \mathrm{a}$ & $413 \mathrm{a}$ & 420def & $1031 \mathrm{a}$ & $1398 \mathrm{a}$ \\
\hline BARImung-5 & $0.51 \mathrm{e}$ & $3.24 \mathrm{de}$ & $7.05 \mathrm{~g}$ & $10.99 \mathrm{gh}$ & $11.20 \mathrm{~g}$ & $273 b c$ & $281 \mathrm{~g}$ & $294 \mathrm{i}$ & $221 \mathrm{~g}$ \\
\hline BARImung-6 & $0.46 \mathrm{e}-\mathrm{h}$ & $2.92 \mathrm{ef}$ & $5.64 \mathrm{i}$ & $10.80 \mathrm{~h}$ & $15.78 \mathrm{f}$ & $206 d$ & $312 \mathrm{~g}$ & $416 \mathrm{~h}$ & $598 \mathrm{e}$ \\
\hline BINAmung-2 & $0.77 \mathrm{a}$ & $3.61 \mathrm{~cd}$ & $8.44 \mathrm{def}$ & $16.97 \mathrm{bc}$ & $30.63 \mathrm{ab}$ & $284 \mathrm{bc}$ & $483 c$ & $853 b$ & $1366 a$ \\
\hline BINAmung-5 & $0.71 \mathrm{~b}$ & $3.30 \mathrm{de}$ & $7.92 \mathrm{f}$ & $17.84 \mathrm{~b}$ & $20.40 \mathrm{e}$ & $259 \mathrm{c}$ & $462 \mathrm{~cd}$ & $592 \mathrm{f}$ & $656 \mathrm{e}$ \\
\hline BINAmung-6 & $0.64 \mathrm{c}$ & $3.23 \mathrm{de}$ & $10.1 \mathrm{~b}$ & $13.37 \mathrm{de}$ & $15.52 \mathrm{f}$ & $259 c$ & $388 \mathrm{f}$ & $426 \mathrm{~h}$ & $415 \mathrm{f}$ \\
\hline BINAmung-7 & 0.50 ef & $2.58 \mathrm{fg}$ & 7.98 ef & $15.63 \mathrm{~cd}$ & $28.42 b c$ & $208 d$ & $540 \mathrm{~b}$ & $765 \mathrm{c}$ & $1279 \mathrm{~b}$ \\
\hline BUmung-1 & $0.44 \mathrm{gh}$ & $2.41 \mathrm{~g}$ & $6.11 \mathrm{hi}$ & $13.52 \mathrm{def}$ & $27.98 b c$ & $167 d$ & $400 \mathrm{ef}$ & $741 \mathrm{~cd}$ & $1445 \mathrm{a}$ \\
\hline BUmung-2 & $0.48 \mathrm{efg}$ & $2.43 \mathrm{~g}$ & $6.53 \mathrm{gh}$ & $12.31 \mathrm{fgh}$ & $16.97 \mathrm{f}$ & $195 d$ & $378 \mathrm{f}$ & $410 \mathrm{~h}$ & $666 \mathrm{e}$ \\
\hline BUmung-4 & $0.45 f g h$ & $3.57 \mathrm{~cd}$ & $8.99 \mathrm{~cd}$ & $13.13 \mathrm{efg}$ & $14.52 \mathrm{f}$ & $312 b$ & 442 cde & $514 \mathrm{~g}$ & $239 \mathrm{~g}$ \\
\hline F-test & $* *$ & $* *$ & $* *$ & $* *$ & $* *$ & $* *$ & $* *$ & $* *$ & $* *$ \\
\hline $\mathrm{CV}(\%)$ & 5.64 & 6.56 & 5.54 & 8.20 & 7.95 & 9.71 & 5.75 & 4.38 & 6.94 \\
\hline
\end{tabular}

In a column, means followed by same letter (s) do not differ significantly at $5 \%$ level by DMRT.

$*$ Significant at $1 \%$ level of probability; DAS = Days after sowing

TDM was slowly increased up to 35 DAS in all the varieties and thereafter increased rapidly up to maturity (Table 2). Result revealed that high LA bearing cultivars produced higher TDM than low LA bearing cultivars. These results indicate that TDM depend on LA in mungbean. The result is consistent with Mondal et al. (2011c) who reported that high TDM accumulation was the result of increase LA in mungbean. The AGR increased with age till 55 DAS in three cultivars, BARImung-5, BINAmung- 6 and BUmung-4 followed by a decline where as other nine cultivars, the AGR increased with age till 65 DAS (Table 2). However, high TDM producing cultivars showed high AGR than low TDM producing ones. 
Pod number, the most important yield attribute, showed significant differences among the varieties (Table 3). Results revealed that bold seeded cultivars produced fewer numbers of pods/plant as well as seed yield/plant. BARImung-2, BARImung-3, BINAmung-2, BINAmung-7 and BUmung-1 are the small seeded cultivars, produced higher number of pods/plant (range 25.033.2) and also showed higher seed yield/plant (range 6.02-6.94 g) except BARImung-4. This result is consistent with Singh et al. (2008) who reported that seed yield had negatively correlated with seed size in mungbean. Harvest index was greater in low yielding cultivars than high yielding ones indicating assimilates partition to economic yield is better in low yielding ones than high yielders.

Table 3. Some yield contributing characters and yield in twelve mungbean varieties.

\begin{tabular}{llllllll}
\hline Varieties & $\begin{array}{c}\text { Pods/ plant } \\
(\text { no. })\end{array}$ & $\begin{array}{c}\text { Pod length } \\
(\mathrm{cm})\end{array}$ & $\begin{array}{c}\text { Seeds/ } \\
\text { pod (no.) })\end{array}$ & $\begin{array}{c}\text { Single pod } \\
\text { weight }(\mathrm{mg})\end{array}$ & $\begin{array}{c}1000 \text {-seed } \\
\text { weight }(\mathrm{g})\end{array}$ & $\begin{array}{c}\text { Seed yield/ } \\
\text { plant }(\mathrm{g})\end{array}$ & $\begin{array}{c}\text { Harvest } \\
\text { index }(\%)\end{array}$ \\
\hline BARImung-2 & $26.6 \mathrm{~b}$ & $6.92 \mathrm{ef}$ & $10.37 \mathrm{abc}$ & $403 \mathrm{~g}$ & $31.9 \mathrm{e}$ & $6.94 \mathrm{a}$ & $18.8 \mathrm{~cd}$ \\
BARImung-3 & $28.6 \mathrm{~b}$ & $6.74 \mathrm{fg}$ & $10.12 \mathrm{bc}$ & $464 \mathrm{f}$ & $37.5 \mathrm{~d}$ & $6.00 \mathrm{bc}$ & $31.8 \mathrm{a}$ \\
BARImung-4 & $25.4 \mathrm{~b}$ & $7.04 \mathrm{e}$ & $10.19 \mathrm{abc}$ & $445 \mathrm{f}$ & $33.9 \mathrm{e}$ & $5.33 \mathrm{de}$ & $14.3 \mathrm{e}$ \\
BARImung-5 & $16.1 \mathrm{~cd}$ & $8.40 \mathrm{~cd}$ & $10.40 \mathrm{abc}$ & $665 \mathrm{~d}$ & $50.4 \mathrm{~b}$ & $5.10 \mathrm{ef}$ & $28.4 \mathrm{~b}$ \\
BARImung-6 & $13.7 \mathrm{~d}$ & $8.98 \mathrm{a}$ & $10.50 \mathrm{abc}$ & $843 \mathrm{a}$ & $55.4 \mathrm{a}$ & $4.68 \mathrm{~cd}$ & $32.6 \mathrm{a}$ \\
BINAmung-2 & $33.2 \mathrm{a}$ & $6.27 \mathrm{~h}$ & $10.22 \mathrm{abc}$ & $323 \mathrm{~h}$ & $27.0 \mathrm{f}$ & $6.02 \mathrm{ef}$ & $16.2 \mathrm{de}$ \\
BINAmung-5 & $18.3 \mathrm{c}$ & $8.23 \mathrm{~d}$ & $10.21 \mathrm{abc}$ & $563 \mathrm{e}$ & $43.8 \mathrm{c}$ & $6.23 \mathrm{~b}$ & $20.0 \mathrm{c}$ \\
BINAmung-6 & $16.4 \mathrm{~cd}$ & $8.72 \mathrm{~b}$ & $10.60 \mathrm{a}$ & $788 \mathrm{~b}$ & $52.8 \mathrm{ab}$ & $5.25 \mathrm{de}$ & $26.9 \mathrm{~b}$ \\
BINAmung-7 & $32.4 \mathrm{a}$ & $6.59 \mathrm{~g}$ & $10.10 \mathrm{bc}$ & $381 \mathrm{~g}$ & $33.3 \mathrm{e}$ & $6.50 \mathrm{ab}$ & $21.3 \mathrm{c}$ \\
BUmung-1 & $25.0 \mathrm{~b}$ & $7.12 \mathrm{e}$ & $10.57 \mathrm{ab}$ & $400 \mathrm{~g}$ & $34.2 \mathrm{e}$ & $5.17 \mathrm{def}$ & $16.0 \mathrm{de}$ \\
BUmung-2 & $14.8 \mathrm{~cd}$ & $8.47 \mathrm{bcd}$ & $10.50 \mathrm{abc}$ & $650 \mathrm{~d}$ & $50.0 \mathrm{~b}$ & $4.68 \mathrm{f}$ & $32.4 \mathrm{a}$ \\
BUmung-4 & $13.8 \mathrm{~d}$ & $8.65 \mathrm{bc}$ & $10.09 \mathrm{c}$ & $703 \mathrm{c}$ & $50.6 \mathrm{~b}$ & $5.00 \mathrm{ef}$ & $27.0 \mathrm{~b}$ \\
\hline F test & $* *$ & $* *$ & $* *$ & $* *$ & $* *$ & $* *$ & $* *$ \\
\hline CV $(\%)$ & 9.98 & 2.00 & 2.35 & 2.85 & 3.85 & 5.11 & 7.16 \\
\hline
\end{tabular}

In a column, means followed by same letter(s) do not differ significantly at 5\% level by DMRT.

**Significant at $1 \%$ level of probability

In case of unit area basis, result revealed that seed yield/ha was greater in low yielding varieties than high yielding ones (except BINAmung-7) at all locations (Table 4) might be due to increase number of plants accommodation per unit area for closer plant spacing $(25 \mathrm{~cm} \times 10 \mathrm{~cm})$. It is possible because of low yielding varieties had lower canopy area than high yielding ones (Table 1). On the other hand, high yielding varieties had higher yield/plant but showed medium seed yield per hectare due to lower number of plants per unit area because of its bushy stature (Table 1). The highest seed yield/ha was recorded in BINAmung-5 (1758 kg/ha) followed by BARImung-6 (1707 kg/ha) with same statistical rank. In contrast, the lowest seed yield/ha was recorded in BUmung-4 (1444 kg/ha).

It may be concluded that high yielding cultivars have higher leaf area as well as TDM and AGR which resulted higher number of pods/plant than the low yielders in mungbean. Though low yielding cultivars showed lower yield/plant but produced higher seed yield per hectare and also matured 10 - 15 days earlier compared to high yielding ones. 
Table 4. Days to maturity and seed yield over six locations of 12 mungbean varieties conducted during Kharif-I season, 2009.

\begin{tabular}{|c|c|c|c|c|c|c|c|c|}
\hline \multirow{3}{*}{ Varieties } & \multirow{3}{*}{$\begin{array}{l}\text { Days to } \\
\text { maturity }\end{array}$} & \multicolumn{7}{|c|}{ Seed yield (kg/ha) } \\
\hline & & \multicolumn{7}{|c|}{ Locations } \\
\hline & & $\begin{array}{l}\text { Mymen- } \\
\text { singh }\end{array}$ & Magura & $\begin{array}{l}\text { Chua- } \\
\text { danga }\end{array}$ & Ishurdi & Natore & Rangpur & $\begin{array}{l}\text { Mean over } \\
\text { locations }\end{array}$ \\
\hline BARImung-2 & $74.3 \mathrm{a}$ & $1562 \mathrm{de}$ & $1543 \mathrm{c}$ & $1583 \mathrm{a}$ & $1803 \mathrm{~d}$ & $2067 \mathrm{c}$ & $1306 \mathrm{c}$ & $1644 \mathrm{~b}$ \\
\hline BARImung-3 & $66.7 \mathrm{~cd}$ & $1701 \mathrm{~b}$ & $1595 \mathrm{ab}$ & $1567 \mathrm{a}$ & $1921 \mathrm{bc}$ & $2089 c$ & $1139 \mathrm{f}$ & $1669 a b$ \\
\hline BARImung-4 & $75.0 \mathrm{a}$ & $1199 \mathrm{~h}$ & $1217 \mathrm{e}$ & $1305 \mathrm{~d}$ & $1912 b c$ & $2278 \mathrm{~b}$ & $1262 \mathrm{~cd}$ & $1529 \mathrm{~cd}$ \\
\hline BARImung-5 & $61.0 \mathrm{e}$ & $1529 \mathrm{de}$ & $1450 \mathrm{~d}$ & $1461 \mathrm{~b}$ & $1870 \mathrm{bc}$ & $2078 \mathrm{c}$ & $1367 \mathrm{~b}$ & $1626 \mathrm{c}$ \\
\hline BARImung-6 & $59.7 \mathrm{e}$ & $1703 \mathrm{c}$ & $1450 \mathrm{~d}$ & $1517 \mathrm{ab}$ & $1874 \mathrm{bc}$ & $2433 \mathrm{ab}$ & $1262 \mathrm{c}$ & $1707 \mathrm{a}$ \\
\hline BINAmung-2 & $74.7 \mathrm{a}$ & $1131 \mathrm{~h}$ & $1207 \mathrm{e}$ & 1139 e & $1847 \mathrm{c}$ & $2017 \mathrm{c}$ & $1322 \mathrm{bc}$ & $1444 \mathrm{~d}$ \\
\hline BINAmung-5 & $68.7 \mathrm{bc}$ & $1869 \mathrm{a}$ & $1562 \mathrm{ab}$ & $1528 \mathrm{ab}$ & $1971 \mathrm{~b}$ & $2267 \mathrm{~b}$ & $1355 \mathrm{~b}$ & $1758 \mathrm{a}$ \\
\hline BINAmung-6 & $61.0 \mathrm{e}$ & $1575 \mathrm{~d}$ & $1647 \mathrm{a}$ & $1417 \mathrm{c}$ & $1744 \mathrm{~d}$ & $2100 \mathrm{c}$ & $1300 \mathrm{c}$ & $1631 \mathrm{c}$ \\
\hline BINAmung-7 & $71.7 \mathrm{ab}$ & $1471 \mathrm{f}$ & $1573 \mathrm{ab}$ & $1156 \mathrm{e}$ & $1716 \mathrm{~d}$ & $2594 \mathrm{a}$ & $1539 a$ & $1675 \mathrm{ab}$ \\
\hline BUmung-1 & $73.3 \mathrm{a}$ & $1163 \mathrm{~h}$ & $1605 a b$ & $1461 \mathrm{~b}$ & $2087 \mathrm{a}$ & $2155 \mathrm{c}$ & $1233 \mathrm{de}$ & $1617 \mathrm{c}$ \\
\hline BUmung-2 & $63.3 \mathrm{~d}$ & $1403 \mathrm{~g}$ & $1583 \mathrm{a}$ & $1417 \mathrm{c}$ & $1555 \mathrm{e}$ & $1878 \mathrm{~d}$ & $1200 \mathrm{e}$ & $1506 \mathrm{~d}$ \\
\hline BUmung-4 & $60.7 \mathrm{e}$ & $1501 \mathrm{ef}$ & $1390 \mathrm{~d}$ & $1333 \mathrm{~d}$ & $1632 \mathrm{e}$ & $1822 \mathrm{~d}$ & $1089 \mathrm{f}$ & $1461 \mathrm{~d}$ \\
\hline F-test & $* *$ & $* *$ & $* *$ & $* *$ & $* *$ & $* *$ & $* *$ & $* *$ \\
\hline $\mathrm{CV}(\%)$ & 3.52 & 2.60 & 5.16 & 3.33 & 8.22 & 10.24 & 5.66 & 5.18 \\
\hline
\end{tabular}

In a column, means followed by same letter (s) do not differ significantly at $5 \%$ level by DMRT.

**Significant at $1 \%$ level of probability.

\section{Acknowledgment}

The authors acknowledge the authority of the Ministry of Science and Information \& Communication Technology, Government of the People's Republic of Bangladesh, for providing financial support to carry out the experiments from the Research and Development Project.

\section{References}

Fakir MSA, Mondal MMA, Ismail MR and Ashrafuzzaman M 2011. Flowering pattern and reproductive efficiency in mungbean. Int. J. Agric. Biol. 13: 966-970.

Hunt R 1978. Plant growth analysis studies in biology. Edward Arnold Ltd. London. pp. 65-67.

Mondal MMA, Fakir MSA, Islam MN and Samad MA 2011c. Physiology of seed yield in mungbean: growth and dry matter production. Bangladesh J. Bot. 40: 133-138.

Mondal MMA, Hakim MA, Juraimi AS and Azad MAK 2011b. Contribution of morpho-physiological attributes in determining yield of mungbean. African J. Biotech. 10: 12897-12904.

Mondal MMA, Rahman MA, Akter MB and Fakir MSA 2011a. Effect of foliar application of nitrogen and micronutrients on growth and yield in mungbean. Legume Res. 34: 166-171.

Pandey RK, Saxena MC and Singh VB 1978. Growth analysis of blackgram genotypes. Indian J. Agric. Sci. 48: $466-473$.

Singh SK, Singh IP, Singh BB and Singh O 2008. Correlation and path coefficient studies for yield and its components in mungbean. Legume Res. 32: 316-318. 